\title{
Catalytic activity of a novel serine/threonine protein phosphatase PP5 from Leishmania major
}

\author{
Brianna Norris-Mullins, Paola Vacchina, and Miguel A. Morales* \\ Department of Biological Sciences, Eck Institute for Global Health, University of Notre Dame, Notre Dame, IN 46556, USA
}

Received 7 March 2014, Accepted 24 May 2014, Published online 4 June 2014

\begin{abstract}
Leishmaniasis is a vector-borne disease caused by protozoan parasites of the genus Leishmania. Our knowledge of protein phosphatases (PPs) and their implication in signaling events is very limited. Here we report the expression, characterization and mutagenesis analysis of a novel protein phosphatase 5 (PP5) in Leishmania major. Recombinant PP5 is a bona fide phosphatase and is enzymatically active. Site-directed mutagenesis revealed autoinhibitory roles of the N-terminal region. This is a rational first approach to understand the role of PP5 in the biology of the parasite better as well as its potential future applicability to anti-parasitic intervention.
\end{abstract}

Key words: signaling, phosphatases, mutagenesis, activity, drug target.

\begin{abstract}
Résumé - Activité catalytique d'une nouvelle phosphatase de protéine sérine/thréonine PP5 de Leishmania major. La leishmaniose est une maladie transmise par un vecteur, due à des parasites protozoaires appartenant au genre Leishmania. Notre connaissance des phosphatases de protéines (PPs) et leur implication dans la signalisation est très limitée. Nous rapportons ici l'expression, la caractérisation et les analyses de mutagenèse d'une nouvelle PP5 chez Leishmania major. La PP5 recombinante est une phosphatase authentique, enzymatiquement active. La mutagenèse dirigée a identifié les rôles auto-inhibiteurs de la région N-terminale de la PP5. Il s'agit d'une première approche rationnelle vers une meilleure compréhension du rôle de la PP5 dans la biologie du parasite, ainsi que pour une utilisation potentielle dans le cadre d'interventions antiparasitaires.
\end{abstract}

\section{Introduction}

Leishmaniasis is a vector-borne disease caused by protozoan parasites of the genus Leishmania [3]. The disease's medical implications vary from disfiguring cutaneous leishmaniasis to life-threatening visceral leishmaniasis. Leishmaniasis is considered a neglected tropical disease with more than 12 million people currently infected and approximately 2 million new cases emerging each year, though these statistics are largely underestimated due to misdiagnosis and lacking surveillance systems [2]. Notably, co-infection with the human immunodeficiency virus (HIV/AIDS) is more detrimental to patients exhibiting disease, as the infections target similar immune cells and vastly promote clinical progressions of each other [1]. Though there is no vaccine for leishmaniasis, some treatments are available and are, usually, species-specific. Currently the most common drugs used to treat infection are pentavalent antimony, amphotericin B, and miltefosine. Unfortunately, these drugs are toxic and expensive. As resistance is a common

\footnotetext{
*Corresponding author: miguel.morales@nd.edu
}

feature of most of them, the urge for newer drugs is imperative [10]. During the infectious cycle, Leishmania differentiates from the extracellular flagellated promastigote to the intracellular pathogenic amastigote form. Promastigotes develop in the midgut of sandflies and following infection in humans, differentiate to intracellular amastigotes that multiply inside the macrophage lysosome [14]. This differentiation is triggered by environmental signals, mainly acidic $\mathrm{pH}$ and high temperature in the mammalian host [24]. Signal transduction cascades often relay these environmental stimuli through reversible phosphorylation via kinases and phosphatases, ultimately leading to changes in protein activity, interaction and expression profiles [11]. Our knowledge of these signaling molecules in Leishmania is scarce despite their essential roles in the biology of the parasite. Mitogen-activated protein kinases (MAPKs) are conserved across virtually all eukaryotic organisms. The importance of the core MAPK pathway has been revealed in Leishmania mexicana and deletion analysis of LmxMPK1 and 2 showed that both are essential for survival in the amastigote stage [21, 22]. LmxMPK3 regulates flagellar length [9] and LmxMKK is the MAPKK responsible for its regulation [23]. 
On the other hand, our understanding of the biology of protein phosphatases in trypanosomatids is poor despite the fact that protein dephosphorylation is largely implicated in critical post-translational modifications [15], differentiation [18, 19], and more recently in parasite drug resistance [4]. Approximately $96 \%-99 \%$ of proteins in eukaryotes are phosphorylated on Serine and Threonine (Ser/Thr) residues. Ser/Thr phosphatases are divided into three families: phosphoprotein phosphatases (PPPs), metallo-dependent protein phosphatases (PPM), and aspartate-based phosphatases with a DxDxT/V motif [17]. Protein phosphatase 5 (PP5) is a putative gene belonging to the PPP family. It has a unique characteristic in that it differs from other phosphatases in the PPP family due to its N-terminal tetratricopeptide repeat (TPR) domains, which are momentous in protein-protein interactions and autoinhibition [5]. In mammalian systems, PP5 TPR domains associate with several proteins that affect signal transduction networks, including the glucocorticoid receptor (GR)-heat shock protein 90 heterocomplex [7]. The catalytic domain of PP5 shares 35\%-45\% sequence identity with the catalytic domains of other PPP phosphatases, including protein phosphatase 1 (PP1), 2A (PP2A), and $2 \mathrm{~B} /$ calcineurin. It is less abundant than the above-mentioned PPPs in mammalian systems and its basal activity is low under typical phosphatase assay conditions [8]. Here we depict the catalytic activity of a novel protein phosphatase PP5 in L. major and dissect the residues implicated in its autoinhibitory regulation.

\section{Materials and methods}

\section{Bioinformatics}

Leishmania major PP5 was used as an initial query for PSIBLAST and sequences corresponding to putative PP5 proteins from the sequenced genomes of Leishmania infantum, L. braziliensis, L. mexicana, Trypanosoma cruzi, T. brucei, Homo sapiens, Mus musculus, Arabidopsis thaliana, Bos taurus, Schistosoma mansoni, and Saccharomyces cerevisae were retrieved using the TriTrypDB and UniProt databases: (http:// tritrypdb.org/tritrypdb/) and (www.uniprot.org). Sequences were aligned with Clustal X (v2.0) and edited with Jalview [20]. Alignments were converted to MEGA compatible files and fed into the MEGA5.2 software package. A NeighborJoining tree was computed with 500 bootstrap replicates.

\section{Molecular constructs}

In order to generate a recombinant PP5 protein, a $1.4 \mathrm{~kb}$ region containing PP5 was amplified from genomic DNA of

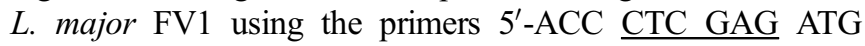
GAG GAG TCC GAC CGC-3' (Xhol), R 5'-GCC GCG GCC GCT TAA AAT AGA CCC GCG CC-3' (NotI) and LongAmp high-fidelity Taq-DNA polymerase (New England Biolabs) following the manufacturer's recommendation. The product was cloned into pGEM-T (Promega) to create pGEM-T-PP5. N-terminal GST-PP5 fusions (Glutathione $\mathrm{S}$-Transferase) were obtained by inserting the $1.4 \mathrm{~kb}$ PP5

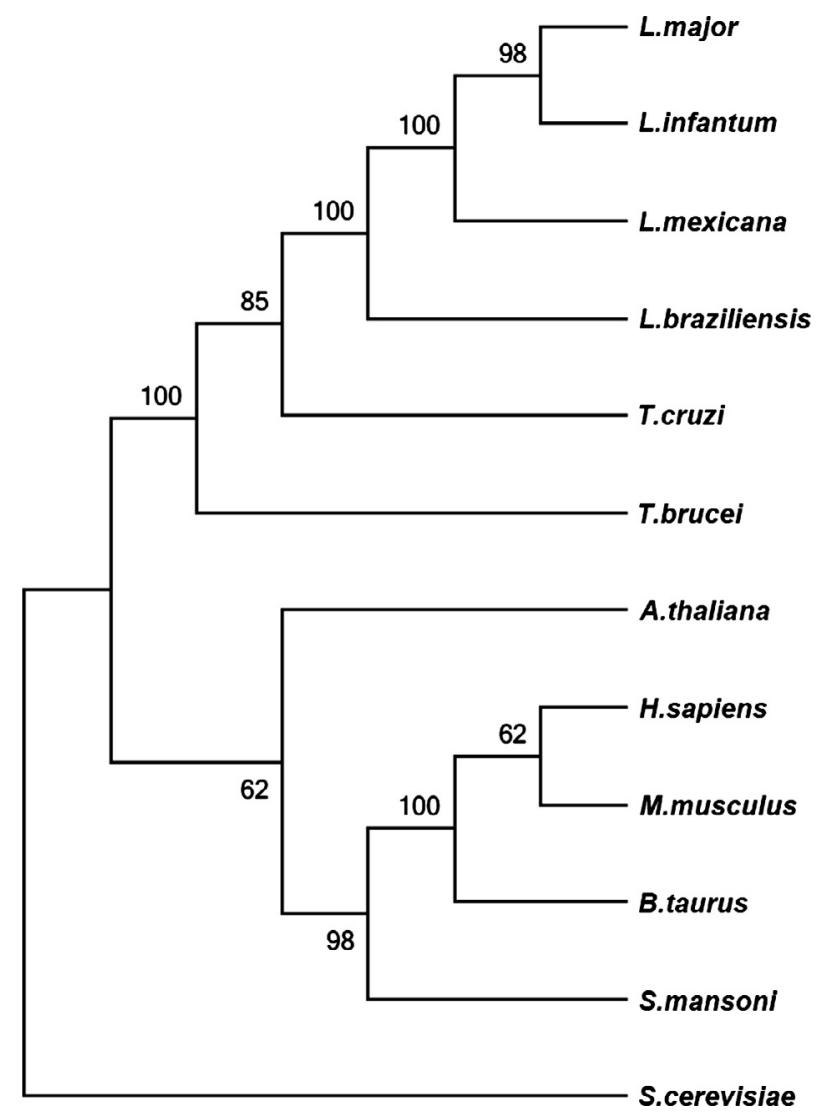

Figure 1. Bioinformatics analysis. The relationship of Leishmania major PP5 (XP_001682421.1) to PP5 homologs of Leishmania infantum (XP_001464832.1), Leishmania mexicana (XP_003874029.1), Leishmania braziliensis (XP_001563942.2), Trypanosoma cruzi (XP_820611.1), Trypanosoma brucei (XP_827850.1), Arabidopsis thaliana (NP_565985.1), Homo sapiens (NP_006238.1), Mus musculus (NP_035285.2), Bos taurus (NP_001179178.1), Schistosoma mansoni (XP_002580099.1) and Saccharomyces cerevisiae (EDN61714.1) was analyzed by multiple alignment and cluster analysis using Clustal X. Alignment was fed into MEGA5.2 software and a Neighbor-Joining tree was computed with 500 bootstrap replicates. Numbers on nodes indicate bootstrap support.

fragment from pGEM-T into the respective site of pGEX-5x. pGEX-5x-PP5 constructs were then transformed in BL-21 Escherichia coli $($ E. coli) cells (NEB). Cells transfected with the empty vector, pGEX-5x, were used as mock controls.

\section{Expression and purification}

Cell lines were grown in LB broth and ampicillin (100 ug/ $\mathrm{mL}$ ). At $\mathrm{OD}=0.5$ the cultures were induced with $0.1 \mathrm{mM}$ isopropyl-1-thio- $\beta$-D-galactopyranoside (IPTG) for $2 \mathrm{~h}$ at room temperature. Cells were harvested and lysed with BugBuster (Novagen). Recombinant proteins were purified with GST-spin columns (GE Healthcare) as per the manufacturers' protocol. Following elution each culture was dialyzed against $50 \mathrm{mM}$ Tris-HCl (pH 8.0). 


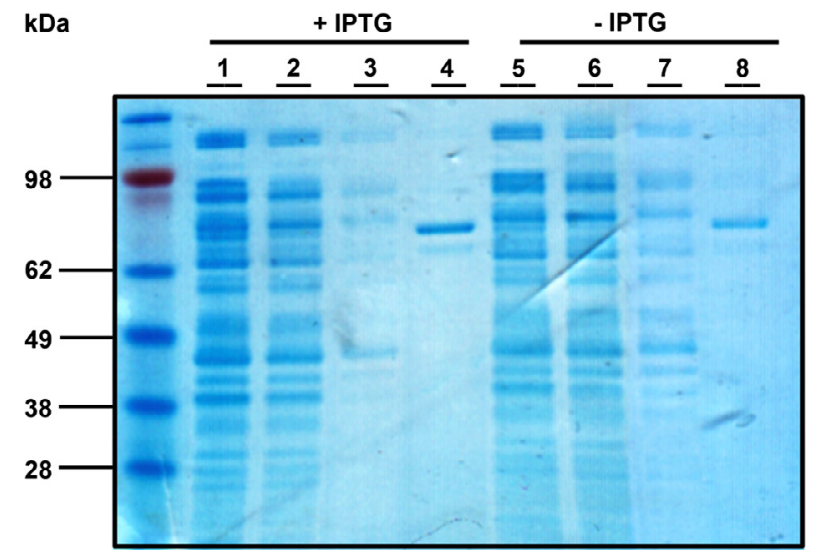

Figure 2. Purification of GST-PP5 from BL-21 cells. Cells were grown to $\mathrm{OD}=0.5$ and IPTG-induced $(0.1 \mathrm{mM})$ (lanes $1-4)$ and uninduced (lanes 5-8) cultures were left to stir for two hours at room temperature. Protein was extracted and purified using GST-spin columns. Lanes are representative of the elution process: total protein lysate (lanes 1 and 5), flow through washes (lanes 2-3 and 6-7) and GST-PP5 protein eluate (lanes 4 and 8).

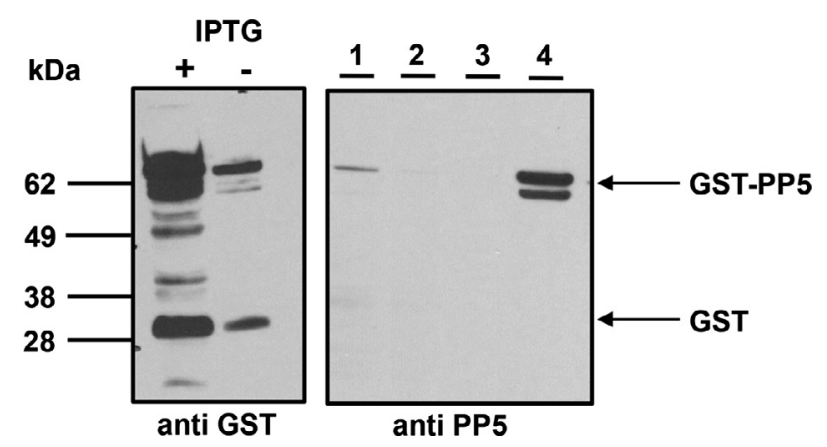

Figure 3. Expression of GST-PP5 in BL-21 cells. Expression of GST-PP5 as detected by Western blotting using an anti-GST antibody (left panel) induced with $0.1 \mathrm{mM}$ IPTG or uninduced. Right panel, polyclonal anti-PP5 antibody. Lanes are representative of the elution process: total protein lysate (lane 1), flow through washes (lanes 2 and 3) and GST-PP5 protein eluate (lane 4). The molecular weight of standard proteins is indicated in $\mathrm{kDa}$.

\section{Western blotting}

Proteins were revealed using the following primary antibodies: rabbit polyclonal anti-GST (Santa Cruz Biotechnology), rabbit polyclonal anti-PP5 (Abcam) and anti-rabbit HRP-conjugated secondary antibodies (Pierce). Cell extracts were separated in $4 \%-12 \%$ Bis-Tris NuPAGE gels (Life) and electroblotted onto PVDF membranes (Pierce).

\section{Site-directed mutagenesis}

GST-PP5 mutants were generated using a QuikChange II XL Site-Directed Mutagenesis Kit (Agilent Technologies) as recommended by the manufacturer. Briefly, primers $5^{\prime}$-CTC CCG CGG CAA CGC CGA GGG ACT CTC G-3' and R 5'-CGA GAG TCC CTC GGC GTT GCC GCG GGA G-3'

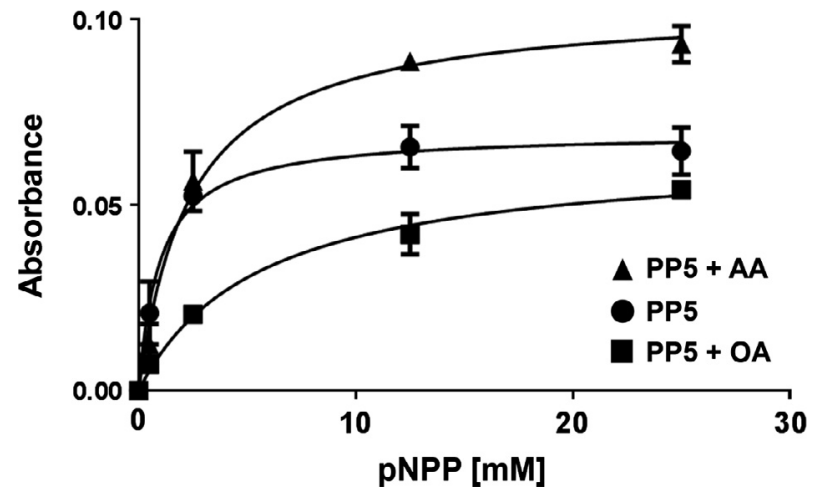

Figure 4. Catalytic activity. PP5 activity was assessed at increasing pNPP substrate concentrations [0] in the presence of $5 \mu \mathrm{M}$ AA [ $[\mathbf{\Delta}]$ or $25 \mu \mathrm{M} \mathrm{OA}[\boldsymbol{-}]$ and absorbance was measured on a plate reader at $405 \mathrm{~nm}$. A dose-dependent non-linear regression curve was generated and standard deviation is shown $(n=3)$.

were used to create a H276A mutation, primers 5'-TAC CTG AAG CTG GCG CTG CCT GGA GCG-3' and R 5'-CGC TCC AGG CAG CGC CAG CTT CAG GTA-3' were used to create an E51A mutation and primers $5^{\prime}$-CGA CCC TGG CTT TGT GGC GGC GTA CTA CCG CAA G-3' and R 5'CTT GCG GTA GTA CGC CGC CAC AAA GCC AGG GTC G-3' were used to create a K72A mutation.

\section{Biochemical characterization}

Activity of L. major recombinant PP5 was measured in a phosphatase assay using p-nitrophenyl phosphate (pNPP) (New England Biolabs) as a substrate. Amounts of recombinant protein were quantified using a Bradford assay. As GST alone can alter protein quantification, samples were further compared with known BSA concentrations using densitometry analysis of a SimplyBlue SafeStain (Invitrogen) stained gel. 1 ug of each recombinant sample, diluted in $1 \times$ colorimetric assay buffer (20 mM Tris pH 7.5, $5 \mathrm{mM} \mathrm{MgCl}_{2}, 1 \mathrm{mM}$ EGTA, $0.02 \% \beta$ mercaptoethanol and $0.1 \mathrm{mg} / \mathrm{mL}$ bovine serum albumin [BSA]), was added to corresponding wells on a 96-well plate and the reaction was initiated by addition of pNPP. After a 45-min incubation at room temperature, $5 \mathrm{~N} \mathrm{NaOH}$ was added to each well to stop the reaction. Absorbance (ABS) was read using a plate reader at $405 \mathrm{~nm}$. Okadaic acid (OA) and arachidonic acid (AA) were purchased from Santa Cruz and Sigma, respectively. Statistical analysis was performed with GraphPad Prism v. 6.04.

\section{Results and discussion}

Using in silico analysis we interrogated the Leishmania major (L. major) genome for protein phosphatase 5 (PP5) (XP_001682421). The amino acid sequence of PP5 in L. major has a 98\% overall identity with PP5 from Leishmania infantum (L. infantum), 77\% with Trypanosma cruzi (T. cruzi), $70 \%$ with Trypanosoma brucei (T. brucei), 50\% with Arabidopsis 


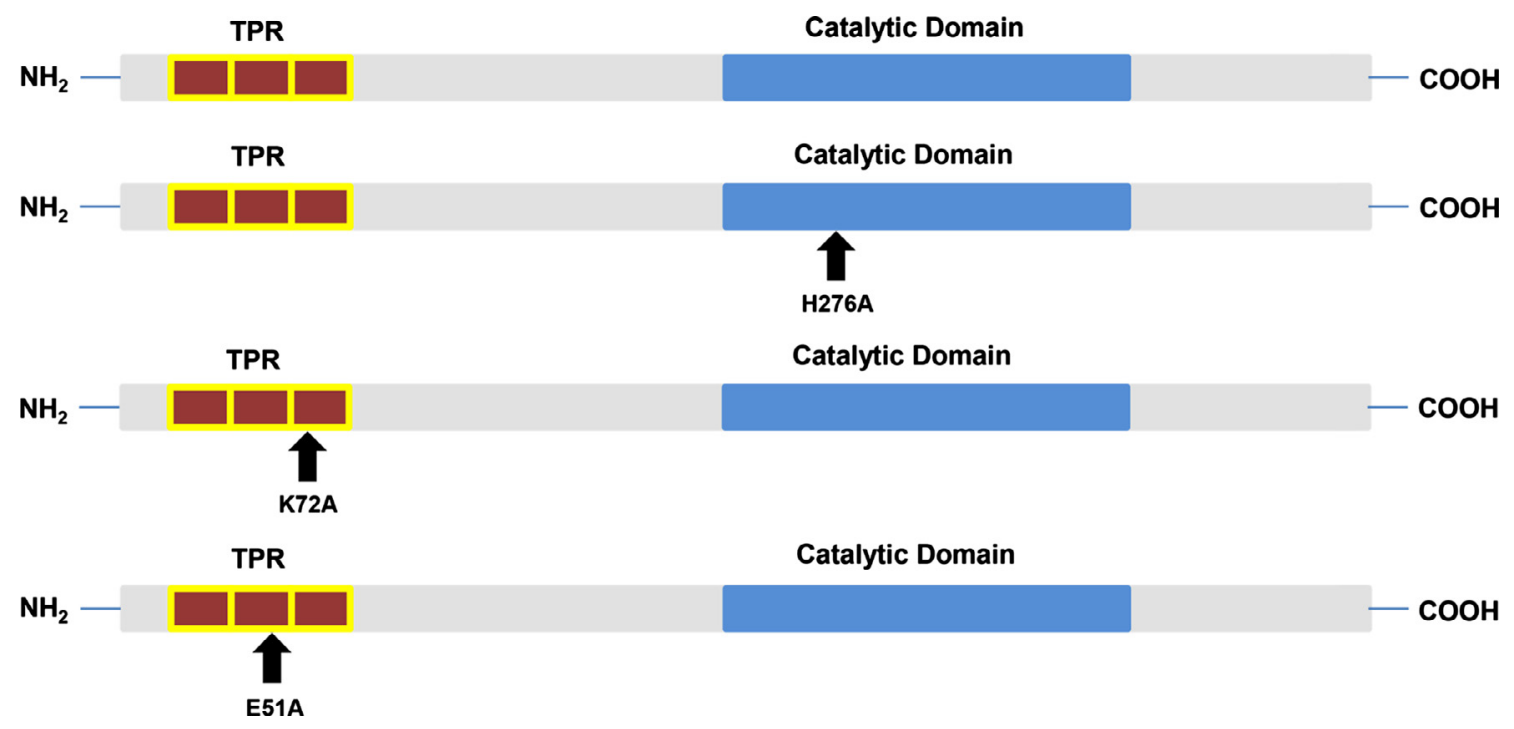

Figure 5. PP5 structure. Leishmania PP5 contains a TPR domain made of three TPRs (red) and one phosphatase catalytic domain (blue). Sitedirected mutagenesis was performed to change Histidine to Alanine at position 276 [H276A], Lysine to Alanine at position 72 [K72A], and Glutamic Acid to Alanine at position 51 [E51A].

thaliana (A. thaliana) and only $46 \%$ with Schistosoma mansoni (S. mansoni), Mus musculus (M. musculus), and Homo sapiens (H. sapiens). L. major PP5 contains three distinct TPR motifs with lower homology to its mammalian counterparts (Fig. S1). Clustering analysis based on multiple alignment confirmed that L. major PP5 is closely related to other trypanosomatid PP5s (Fig. 1).

In order to biochemically characterize PP5 we generated a recombinant GST-PP5 fusion protein which was expressed in BL21 E. coli cells. GST-PP5 expression was increased in IPTG-induced cultures as observed by Coomassie staining (Fig. 2, lanes 4 and 8) and Western blot (Fig. 3, left panel) using a rabbit polyclonal anti-GST antibody. To further test the specificity of GST purification, eluted recombinant proteins were separated, electro-blotted onto PVDF membranes and detected with a polyclonal anti-PP5 antibody (Fig. 3, right panel). Prior to performing the activity assay, a gel was run and stained with Coomassie to compare each sample with BSA loading controls via densitometry (Fig. S2-B). This ensured the use of proper amounts of sample for the activity assay, as GST alone could alter protein quantification (Fig. S2-A).

Being the first description of PP5 in L. major, we measured the catalytic activity of PP5 using increasing concentrations of p-nitrophenyl phosphate (pNPP) as a substrate. A dose-dependent non-linear regression curve was plotted for the activity of PP5 and PP5 in the presence of $5 \mu \mathrm{M}$ arachidonic acid (AA) and $25 \mu \mathrm{M}$ okadaic acid (OA), a generic phosphatase inhibitor (Fig. 4). L. major recombinant PP5 is enzymatically active and is inhibited in the presence of OA. Polyunsaturated fatty acids such as AA interact with the TPR domain of PP5, thus activating the enzyme. Our results are in good accordance with the activation of T. brucei PP5 in the presence of AA and the regulatory effect of the TPR domain on enzymatic activity [6].

As mentioned above, the TPR domain on the N-terminal end of PP5 has a known autoinhibitory function [16], therefore we performed site-directed mutagenesis to test the potential
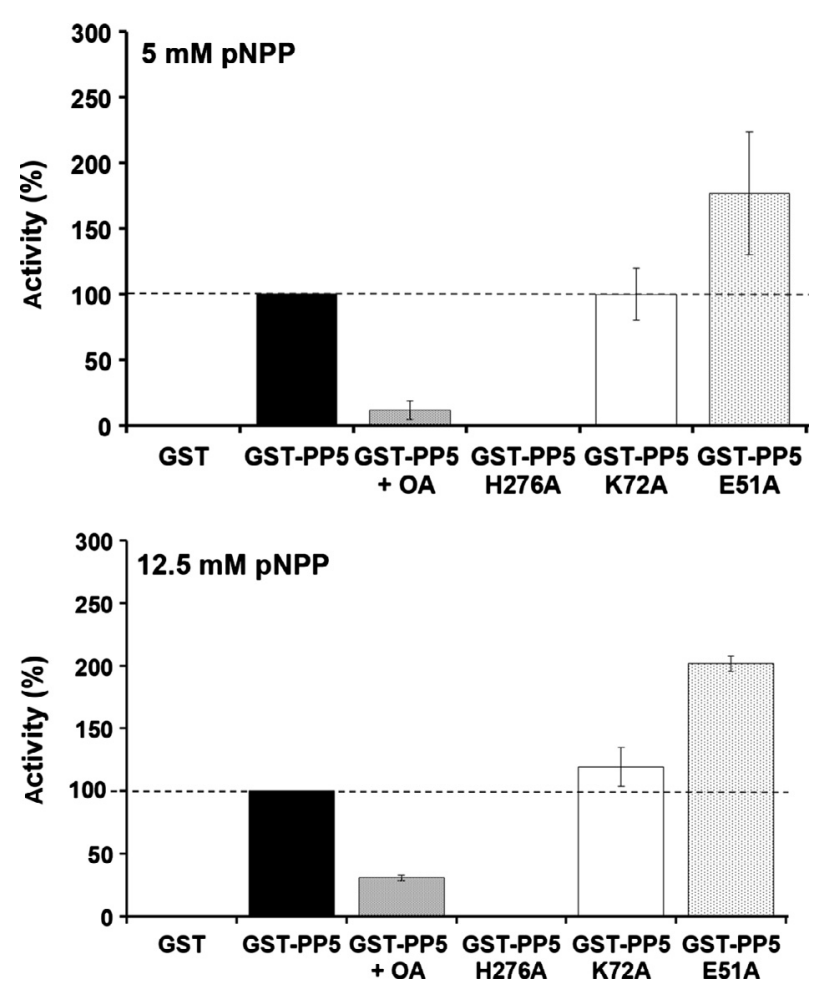

Figure 6. Biochemical characterization of GST-PP5 mutants. Phosphatase activity was assessed at two different pNPP substrate concentrations simultaneously ( $5 \mathrm{mM}$ and $12.5 \mathrm{mM}$, respectively) and absorbance was measured on a plate reader at $405 \mathrm{~nm}$. Standard deviation is denoted by the bars shown $(n=3)$.

negative regulation of a glutamic acid residue (E51A) previously analyzed in mammalian systems [13]. We also mutated a lysine (K72A) residue known to bind to HSP90 [13] and a histidine (H276A) residue known to positively regulate the activity of PP5 [5] (Fig. 5). L. major PP5 is active in the 
presence of $5 \mathrm{mM}$ and $12.5 \mathrm{mM}$ pNPP. $25 \mu \mathrm{M}$ OA was used as a negative control, although complete inhibition was not observed. Glutathione S-Transferase (GST) was assayed as a negative and background control (Fig. 6). H276A mutation on the C-terminal abolishes PP5 activity in good accordance with its mammalian counterpart [5]. As expected, E51A mutation on the TPR domain results in significantly increased activity, regardless of the different pNPP concentrations assayed, suggesting a potential inhibitory role. K72A mutation, which is part of the HSP90-binding groove in the TPR domain [13], has no regulatory function in the catalytic activity of $L$. major PP5. Together these results suggest that L. major PP5 is active and is a bona fide phosphatase.

The autoinhibitory function of the TPR domain is conserved in Leishmania and we found Glu51 to be implicated in this regulation. On the other hand, Lys 72 does not negatively regulate the catalytic activity of PP5; however, further studies using endogenous PP5 parasite extracts are necessary to reveal its implication in the potential interaction with HSP90. Protein binding assays with immunoprecipitated recombinant GST-PP5 and total extracts of $L$. major did not reveal interaction with HSP90 (data not shown). It is important to note that our inability to reveal a PP5- HSP90 interaction may be due to the steric hindrance of GST fused N-terminally to PP5, where the TPR domain is located. Thus, we can only speculate that endogenous L. major PP5 may interact with HSP90. Additionally, it has been shown previously in the related T. brucei that PP5 interacts with and is required to regulate HSP90 function under stress conditions [12].

\section{Conclusions}

The complete genomes of trypanosomatids (www. trytrip.org) have made possible the screening of putative protein phosphatases. The biochemical characterization of this novel PP5 in Leishmania major is a rational first approach to further understand the regulation of signal transduction pathways in this parasite. Additional studies are required to assess substrate specificity and in vivo function. Nonetheless, L. major PP5 shows low similarity with its mammalian counterparts, suggesting it may be targeted for therapeutic intervention.

Acknowledgements. This work was supported by the Eck Institute of Global Health and capitalization funds from the University of NotreDame to MAM. All authors declare no financial/commercial conflict of interest.

\section{References}

1. Alvar J, Aparicio P, Aseffa A, Den Boer M, Canavate C, Dedet JP, Gradoni L, Ter Horst R, Lopez-Velez R, Moreno J. 2008. The relationship between leishmaniasis and AIDS: the second 10 years. Clinical Microbiology Reviews, 21, 334-359.

2. Alvar J, Velez ID, Bern C, Herrero M, Desjeux P, Cano J, Jannin J, den Boer M, the WHO Leishmaniasis Control Team. 2012. Leishmaniasis worldwide and global estimates of its incidence. PloS One, 7, e35671.
3. Ambit A, Woods KL, Cull B, Coombs GH, Mottram JC. 2011. Morphological events during the cell cycle of Leishmania major. Eukaryotic Cell, 10, 1429-1438.

4. Bhandari V, Sundar S, Dujardin JC, Salotra P. 2014. Elucidation of cellular mechanisms involved in experimental paromomycin resistance in Leishmania donovani. Antimicrobial Agents and Chemotherapy, 58, 2580-2585.

5. Borthwick EB, Zeke T, Prescott AR, Cohen PT. 2001. Nuclear localization of protein phosphatase 5 is dependent on the carboxy-terminal region. FEBS Letters, 491, 279-284.

6. Chaudhuri M. 2001. Cloning and characterization of a novel serine/threonine protein phosphatase type 5 from Trypanosoma brucei. Gene, 266, 1-13.

7. Chen MS, Silverstein AM, Pratt WB, Chinkers M. 1996. The tetratricopeptide repeat domain of protein phosphatase 5 mediates binding to glucocorticoid receptor heterocomplexes and acts as a dominant negative mutant. Journal of Biological Chemistry, 271, 32315-32320.

8. Chinkers M. 2001. Protein phosphatase 5 in signal transduction. Trends in Endocrinology and Metabolism, 12, 28-32.

9. Erdmann M, Scholz A, Melzer IM, Schmetz C, Wiese M. 2006. Interacting protein kinases involved in the regulation of flagellar length. Molecular Biology of the Cell, 17, 2035-2045.

10. Garcia-Hernandez R, Manzano JI, Castanys S, Gamarro F. 2012. Leishmania donovani develops resistance to drug combinations. PLoS Neglected Tropical Diseases, 6, e1974.

11. Hunter T. 2000. Signaling -2000 and beyond. Cell, 100, 113-127.

12. Jones C, Anderson S, Singha UK, Chaudhuri M. 2008. Protein phosphatase 5 is required for Hsp90 function during proteotoxic stresses in Trypanosoma brucei. Parasitology Research, 102, 835-844.

13. Kang H, Sayner SL, Gross KL, Russell LC, Chinkers M. 2001. Identification of amino acids in the tetratricopeptide repeat and C-terminal domains of protein phosphatase 5 involved in autoinhibition and lipid activation. Biochemistry, 40, 10485-10490.

14. McConville MJ, Naderer T. 2011. Metabolic pathways required for the intracellular survival of Leishmania. Annual Review of Microbiology, 65, 543-561.

15. Morales MA, Watanabe R, Dacher M, Chafey P, Osorio y Fortea J, Scott DA, Beverley SM, Ommen G, Clos J, Hem S, Lenormand P, Rousselle JC, Namane A, Spath GF. 2010. Phosphoproteome dynamics reveal heat-shock protein complexes specific to the Leishmania donovani infectious stage. Proceedings of the National Academy of Sciences of the United States of America, 107, 8381-8386.

16. Swingle MR, Honkanen RE, Ciszak EM. 2004. Structural basis for the catalytic activity of human serine/threonine protein phosphatase-5. Journal of Biological Chemistry, 279, 3399233999.

17. Szoor B. 2010. Trypanosomatid protein phosphatases. Molecular and Biochemical Parasitology, 173, 53-63.

18. Szoor B, Dyer NA, Ruberto I, Acosta-Serrano A, Matthews KR. 2013. Independent pathways can transduce the life-cycle differentiation signal in Trypanosoma brucei. PLoS Pathogens, 9, e1003689.

19. Szoor B, Ruberto I, Burchmore R, Matthews KR. 2010. A novel phosphatase cascade regulates differentiation in Trypanosoma brucei via a glycosomal signaling pathway. Genes \& Development, 24, 1306-1316. 
20. Waterhouse AM, Procter JB, Martin DM, Clamp M, Barton GJ. 2009. Jalview Version 2 - a multiple sequence alignment editor and analysis workbench. Bioinformatics, 25, 1189-1191.

21. Wiese M. 1998. A mitogen-activated protein (MAP) kinase homologue of Leishmania mexicana is essential for parasite survival in the infected host. EMBO Journal, 17, 2619-2628.

22. Wiese M. 2007. Leishmania MAP kinases - Familiar proteins in an unusual context. International Journal for Parasitology, 37, 1053-1062.
23. Wiese M, Kuhn D, Grunfelder CG. 2003. Protein kinase involved in flagellar-length control. Eukaryotic Cell, 2, 769-777.

24. Zilberstein D, Shapira M. 1994. The role of $\mathrm{pH}$ and temperature in the development of Leishmania parasites. Annual Review of Microbiology, 48, 449-470.

Cite this article as: Norris-Mullins B, Vacchina P \& Morales MA: Catalytic activity of a novel serine/threonine protein phosphatase PP5 from Leishmania major. Parasite, 2014, 21, 25.

\section{- PARASTE}

An international open-access, peer-reviewed, online journal publishing high quality papers on all aspects of human and animal parasitology

Reviews, articles and short notes may be submitted. Fields include, but are not limited to: general, medical and veterinary parasitology; morphology, including ultrastructure; parasite systematics, including entomology, acarology, helminthology and protistology, and molecular analyses; molecular biology and biochemistry; immunology of parasitic diseases; host-parasite relationships; ecology and life history of parasites; epidemiology; therapeutics; new diagnostic tools.

All papers in Parasite are published in English. Manuscripts should have a broad interest and must not have been published or submitted elsewhere. No limit is imposed on the length of manuscripts.

Parasite (open-access) continues Parasite (print and online editions, 1994-2012) and Annales de Parasitologie Humaine et Comparée (1923-1993) and is the official journal of the Société Française de Parasitologie. 